\title{
Understanding the Mentoring Environment Through Thematic Analysis of the Learning Environment in Medical Education: a Systematic Review
}

\author{
Jia Min Hee, MBBS ${ }^{1,2}$, Hong Wei Yap 2,3, Zheng Xuan Ong, MBBS ${ }^{2,4}$, Simone Qian Min Quek, MBBS 2,5 , \\ Ying Pin Toh, MBBS, MRCPCH ${ }^{2,6}$, Stephen Mason, PhD, PGCHE, BA(Hons) ${ }^{7}$, and \\ Lalit Kumar Radha Krishna, MBChB, FRCP, FAMS, MA (Ethics), MA (Med Edu), PhD (Med Ethics) ${ }^{7,2,7,8,9}$
}

\begin{abstract}
YYong Loo Lin School of Medicine, National University of Singapore, Singapore, Singapore; ${ }^{2}$ National Cancer Centre Singapore, Singapore, Singapore; ${ }^{3}$ Lee Kong Chian School of Medicine, Singapore, Singapore; ${ }^{4}$ Singapore General Hospital, Singapore, Singapore; ${ }^{5} \mathrm{Changi}$ General Hospital, Singapore, Singapore; ${ }^{6}$ Department of Family Medicine, National University Hospital, Singapore, Singapore; ${ }^{7}$ University of Liverpool, Liverpool, UK; ${ }^{8}$ Duke-NUS Medical School, Singapore, Singapore; ${ }^{9}$ Centre of Biomedical Ethics, National University of Singapore, Singapore, Singapore.
\end{abstract}

BACKGROUND: Mentoring's success has been attributed to individualised matching, holistic mentoring relationships (MRs) and personalised mentoring environments (MEs). Whilst there is growing data on matching and MRs, a dearth of ME data has hindered development of mentoring programme. Inspired by studies likening MEs to learning environments (LEs) and data highlighting common characteristics between the two, this systematic review scrutinises reports on LEs to extrapolate the findings to the $\mathrm{ME}$ context to provide a better understanding of $\mathrm{ME}$ and their role in the mentoring process.

METHODS: Using identical search strategies, 6 reviewers carried out independent literature reviews of LEs in clinical medicine published between 1 January 2000 and 31 December 2015 using PubMed, ERIC, Cochrane Database of Systematic Reviews, Google Scholar and Scopus databases. Braun and Clarke's (2006) approach to thematic analysis was adopted to circumnavigate LE's evolving, context-specific, goal-sensitive, learner-tutor relationally dependent nature.

RESULTS: A total of 4574 abstracts were identified, 90 articles were reviewed, and 58 full-text articles were thematically analysed. The two themes identified were LE structure and LE culture. LE structure regards the framework that guides interactions within the LE. LE culture concerns the values and practices influencing learnertutor-host organisation interactions.

DISCUSSION: LE is the product of culture and structure that influence and are influenced by the tutor-learner-host organisation relationship. LE structure guides the evolving tutor-learner-host organisation relationship whilst the LE culture nurtures it and oversees the LE structure. Similarities between LEs and MEs allow LE data to inform programme designers of ME's role in mentoring's success.

Electronic supplementary material The online version of this article (https://doi.org/10.1007/s11606-019-05000-y) contains supplementary material, which is available to authorized users.

Received July 7, 2018

Revised November 13, 2018

Accepted February 6, 2019

Published online April 22, 2019
KEY WORDS: learning environment; medical education; medical training; mentoring; mentoring environment.

$\mathrm{J}$ Gen Intern Med 34(10):2190-9

DOI: $10.1007 / \mathrm{s} 11606-019-05000-y$

() Society of General Internal Medicine 2019

\section{INTRODUCTION}

Mentoring shapes professional identities, moulds "values and beliefs" and nurtures professional and personal development through the provision of personalised, appropriate, specific and timely support. ${ }^{1,2}$ Providing such individualised and holistic support depends upon an effective matching process, a nurturing mentoring relationship and a personalised mentoring environment. ${ }^{3-9}$ The matching process involves pairing mentors with like-minded mentees with similar goals, values, beliefs and complementary characteristics to initiate personalised ties that will form the basis for effective mentoring relationships (MRs). ${ }^{2,},{ }^{3}{ }^{10}$ Successful MRs underpin mentoring's success ${ }^{3-9}$ and pivot upon the quality of the ties between mentee, mentor and the host organisation. Nurturing enduring MRs that flourish in changing mentoring conditions require the presence of mentoring environments (MEs). ${ }^{5,7-9,11,12}$

However, unlike matching and MRs which are increasingly the focus of medical education research, ${ }^{5-9,13-17}$ MEs remain poorly studied. $^{3-9,12,18,19}$ Whilst envisaged as an "emotionally safe", supportive and "protégé-centred" setting that facilitates the formation of personalised MRs, ${ }^{5,7-9}$ a formal definition of MEs continues to elude mentoring practice. $^{1-4,11,12,14,18,20-28}$ This lacuna has hindered development of effective mentoring programmes within medical training. ${ }^{5,7-9}$ Inspired by studies that liken MEs to learning environments (LEs) and data highlighting common characteristics within MEs and LEs, this systematic review scrutinises LEs to better understand MEs and guide design and oversight of mentoring programmes. ${ }^{3,4}$, 18, 27, 29-32 


\section{Mentoring Environment}

Davis and Nakamura (2010, p. 1060) characterise ME as a "function of a relationship that rests upon a set of interactional foundations that allow a protégé to capitalize on the strengths of the mentor, and it facilitates behaviors that will enable the protégé to develop and internalize the requisite knowledge, skills, and attitudes (KSAs) as fully as possible". ${ }^{20}$ Sng et al.'s (2017) delineation of the influential role of the mentor and host organisation within the mentoring relationships ${ }^{3,12}$ and Tan et al.'s (2018) description of mentoring's evolving, entwined, goal-specific, context-sensitive, mentor-, mentee-, relational- and organisational-dependent nature (henceforth mentoring's nature), ${ }^{3}, 4,12,18,27$ which are not featured in Davis and Nakamura's (2010) definition, have cast doubts about its applicability in modern mentoring practice. ${ }^{3,4,8,18,20}$ In addition, "over-reliance on cross-sectional designs and self-report data, a failure to differentiate between different forms of mentoring (e.g., formal versus informal), lack of dyadic data, and the use of psychometrically questionable measures" 28 at the heart of Davis and Nakamura's (2010, p. 1060) definition raise further concerns about their characterisation of MEs.

In the absence of robust studies of MEs, Pololi (2002) $)^{29}$ and Kalén, Ponzer and Silén (2012) ${ }^{30}$ suggest that MEs may be understood through studies of learning environments (LEs). ${ }^{3}$, 4, 18, 27, 31, 32 Data that likens LEs and MEs to personalised educational environments ${ }^{31,33}$ that are influenced by the prevailing healthcare and education systems and the professional and personal factors impacting the learner, the tutor and the host organisation add traction to efforts understand MEs through the study of LEs. ${ }^{3,4,}$, 8, 12, 18, 19

\section{Learning Environments}

The World Federation for Medical Education (1998, p. 553) 32 $^{32}$ lists LEs as one of the targets for "the evaluation of medical education programmes" and the UK Standing Committee on Postgraduate Education $(1991)^{34}$ stated that LEs were pivotal to "a working environment that is conducive to learning".

Prevailing data suggest that LEs are shaped by four considerations: one, the setting, context and the goals of the learning process; ${ }^{31,33,35}$ two, the character, abilities, needs and motivations of the learner and the tutor; ${ }^{33,36}$ three, the influence and support of the host organisation which provides financial and administrative support to the tutors, learners and the programme itself; ${ }^{35,37-42}$ four, the impact of external factors such as the social, cultural, healthcare and educational factors within the learning context. $^{31,35}$ These considerations suggest that LEs like MEs exhibit ${ }^{5,7-9}$ context-specific, goal-sensitive, learner-, tutor-, host organisation- and relational-dependent characteristics (henceforth LE's nature). Similarities in the nature and roles of LEs and MEs suggest that data accrued from studies of LEs may be applied to MEs. ${ }^{29,} 30$

\section{METHODOLOGY}

A systematic review of LEs in undergraduate and postgraduate medical schools was conducted in accordance with the PRISMA guidelines. ${ }^{43}$ In the absence of an a priori framework for LEs and MEs, growing suggestions that LEs like MEs are "social phenomena" and LE's nature which restrict studies of LE to comparable learning approaches and settings, a qualitative approach to analysis was adopted. $3,4,18,27,38,40-42,44,45$ Braun and Clarke's (2006) approach to thematic analysis was selected given its use in recent mentoring reviews. ${ }^{5,-9}$ Use of Braun and Clarke's (2006) approach to thematic analysis was also supported by the presence of a wide range of research methodologies amongst the papers reviewed that makes statistical pooling and analysis difficult. ${ }^{3,4}, 18,27,38,40-42,44,45$

Six authors experienced in the use of Braun and Clarke's (2006) approach to thematic analysis carried out independent analyses of the same 10 included articles. Codes were constructed from the "surface" meaning of the data with line-byline coding, proceeding to focused coding, and semantic themes were then identified from the categorisation of these "detail rich" codes. The 10 coded scripts were discussed, and a common coding framework and codebook were agreed upon using the "negotiated consensual validation" approach. ${ }^{1}$ The six authors (HJM, YHW, OZX, SQ, TYP, LK) carried out independent thematic analyses of the remaining articles based on the codebook with new codes discussed online and face-toface at authors' meetings.

The narrative produced was guided by the Best Evidence Medical Education (BEME) Collaboration guide ${ }^{46}$ and the STORIES (Structured approach to the Reporting In healthcare education of Evidence Synthesis) statement. ${ }^{47}$

\section{Literature Search}

The inclusion and exclusion criteria for this review were in accordance with PICOS strategy. The review was designed by the six authors, two librarians and three clinicians and medical education experts. Studies were confined to accounts of mentoring programmes published after 2000 , given the tendency of articles published prior to 2000 to conflate mentoring with supervision, coaching, role modelling and tutoring. ${ }^{3,}$, 7-9 Only articles published in English or had English translations published between 2000 and 2015 were included.

\section{PICOS STRATEGY}

\section{Participants}

Studies were confined to undergraduate and postgraduate medical school programmes in the clinical discipline of general medicine in acknowledgement of LE's nature that prevents comparisons of LEs across heterogeneous educational goals, processes and settings evident in different clinical specialities. Non-medical fields such as midwifery, nursing, psychology, military medicine, complementary medicine, 
paediatrics, obstetrics and gynaecology, service quality, quality improvement, medical simulation, chiropractice, athletic/ sports medicine, surgery and urology were excluded.

\section{Interventions}

Studies examining the "learning environment", "educational environment" or "clinical teaching environment" were included. Studies involving outcome measures, e.g. assessment of the educational environment through validated tools, e.g. DREEM, PHEEM and ACLEEM, were also included.

\section{Outcome}

Articles that studied outcomes of learning environments were selected.

Study Design. Mentoring literature is heterogeneous. As a result, literature reviews, observational studies (both qualitative and quantitative) and interventional studies were included given that the settings and study populations are clear. Perspective pieces, personal accounts, reflections, opinions, commentaries and editorials were excluded to focus this review upon specific practices in acknowledgement of LE's nature.

\section{Search Strategy}

Guided by two librarians, local education experts and clinicians, the six authors (HJM, YHW, OZX, SQ, TYP, LK) designed and carried out independent searches based on agreed upon inclusion and exclusion criteria (refer to Table 1). Using the MeSH terms "Clinical medicine", "Clinical teaching environment" and "Education environment" in the PubMed, ERIC, Cochrane Database of
Systematic Reviews, Google Scholar and Scopus databases, the six authors reviewed articles published in English or had English translations between 1 January 2000 and 31 December 2015. General terms were used to better capture a wide spectrum of accounts of LEs. The searches were carried out between 12 January 2018 and 20 January 2018. The six authors screened the titles and abstracts independently to identify relevant studies meeting the pre-specified inclusion criteria. The authors discussed and agreed upon the list of full-text articles to be reviewed using Sambunjak et al.'s $(2009, \text { p. } 73)^{1}$ "negotiated consensual validation". The authors then screened the list of full text independently and employed Sambunjak et al.'s $(2009, \text { p. } 73)^{1}$ "negotiated consensual validation" to achieve consensus upon the final list of articles to be included in the study at a face-to-face meeting.

\section{Quality Assessment of Studies}

Two authors (HJM, YHW) carried out individual appraisals of quantitative studies using the Medical Education Research Study Quality Instrument (MERSQI) and the Consolidated Criteria for Reporting Qualitative Studies (COREQ) to evaluate the quality and qualitative studies included in this review (refer to Appendix 1 online). The six-person study team met face-to-face to reconcile any differences in their assessments and to forward a consensus-based appraisal of the included studies.

\section{Risk of Bias}

The included studies tended to focus upon positive aspects of LEs leaving the negative accounts of LEs under-represented in the literature.

Table 1 Number of Hits Obtained in PubMed Database

\begin{tabular}{|c|c|c|}
\hline \multicolumn{3}{|c|}{ Recent queries in PubMed } \\
\hline Search & Query & $\begin{array}{l}\text { Items } \\
\text { found }\end{array}$ \\
\hline$\# 1$ & $\begin{array}{l}\text { Search ((("clinical medicine"[MeSH Terms] OR ("clinical"[All Fields] AND "medicine"[All Fields]) OR "clinical } \\
\text { medicine"[All Fields]))) Sort by: Best Match Filters: Publication date from 2000/01/01 to 2015/12/31; English }\end{array}$ & 689,190 \\
\hline$\# 2$ & $\begin{array}{l}\text { Search ((('"education"[Subheading] OR "education"[All Fields] OR "educational status"[MeSH Terms] OR } \\
\text { ("educational"[All Fields] AND "status"[All Fields]) OR "educational status"[All Fields] OR "education"[All Fields] OR } \\
\text { "education"[MeSH Terms])) Sort by: Best Match Filters: Publication date from 2000/01/01 to 2015/12/31; English }\end{array}$ & 603,909 \\
\hline \#3 & 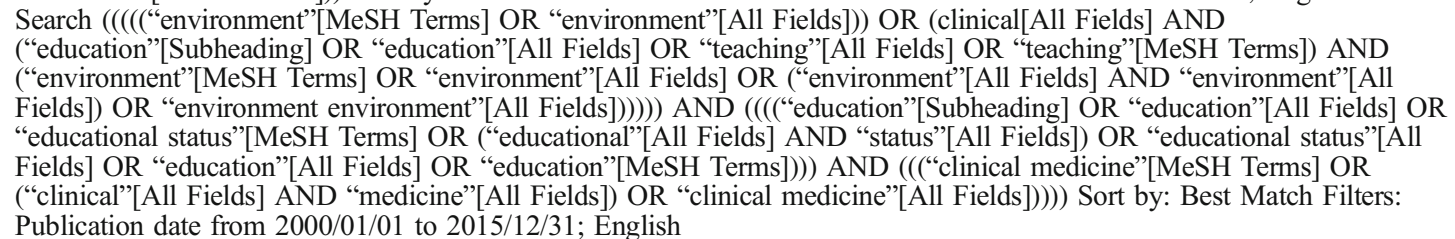 & 3365 \\
\hline \#4 & 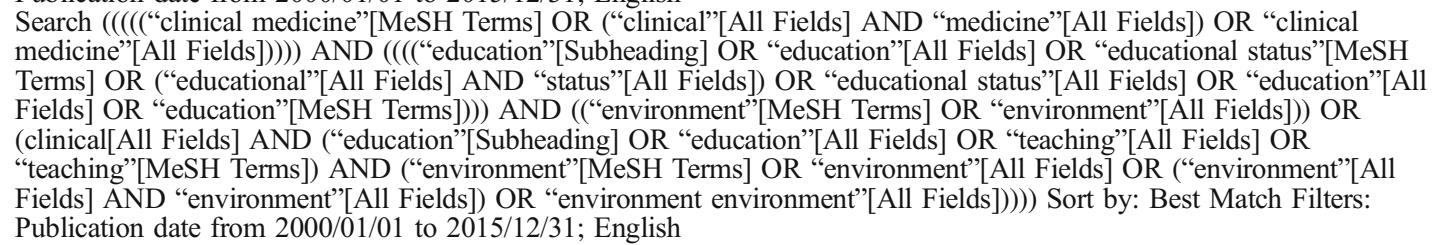 & 3750 \\
\hline
\end{tabular}




\section{RESULTS}

Using Sambunjak et al.'s (2009, p. 73) $)^{1}$ "negotiated consensual validation", the six reviewers identified 4574 abstracts, reviewed 90 full-text articles and included 58 articles ${ }^{36,48-104}$ in this review (Fig. 1). A summary table of the selected articles are included in Appendix 1 online.

The two themes identified were LE structure and LE culture. The structure of LEs (LE structure) concerns the framework and infrastructure that facilitates interactions within the LE. The culture within LEs (LE culture) relates to the norms, values, beliefs, practices and support moulding the socioemotional environment in which learning occurs and that influence interactions between the learner, tutor and organisation. ${ }^{105}$ The term "tutor" encapsulates the terms teacher, tutor, mentor and/ or supervisor featured in the included articles.

\section{LE Structure}

The LE structure refers to the framework that shapes the learning approach and ensures consistent professional and personal support for learners and tutors within the programme. The LE structure is made up of five sub-themes that include the formal curriculum, the host organisation, the tutor, the learner and the learning relationship (relationship between learner and tutor). The formal curriculum is defined as "the actual course of study, the planned content, teaching, evaluation methods, syllabi, and other materials used in any educational setting from lecture halls to labs to seminar rooms. Also included are formal policy statements, regulations, expectations, and competencies for every educational cohort conceivable". ${ }^{106}$

Formal Curriculum. Seventeen articles ${ }^{36,53,56,58,65, ~ 68, ~ 78, ~ 82, ~}$ $87,90-93,99,102,104,107$ discussed the role of the formal curriculum in the LE structure. The formal curriculum maps out the goals, learning objectives, assessment methods and the educational approach employed. ${ }^{58,65,87,104}$ It also specifies the roles and responsibilities of the tutor and learner, the codes of conduct and standards of practice that will be employed. ${ }^{56}$, 99, 102, 107 The formal curriculum stipulates the frequency, duration and timings of meetings, online interactions, tutorials, teaching sessions and feedback sessions ${ }^{53,68,87}$ and the provision of "protected" time for learners and tutors. ${ }^{53,90,91}$ The formal curriculum is thus responsible for structuring the learning process and formal interactions within the LE. ${ }^{36,56,78, ~ 82, ~ 91-93, ~ 99, ~} 107$

Design and content of the formal curriculum is influenced by the host organisation and external factors such as the healthcare and education systems as well as the setting, funding, support and sustainability of the programme, highlighting a reciprocal relationship between the LE structure and the formal curriculum. ${ }^{36,56,78,82,91-93,99,107}$

Host Organisation. A reciprocal relationship is between the host organisation and the LE structure that is also evident in the 12 papers discussing the role of the host organisation. ${ }^{51,53 \text {, }}$
$62,68,77,79,80,85,86,90,91,102,104$ The host organisation is responsible for supporting and overseeing activities stipulated within the curriculum. The host organisation has direct and

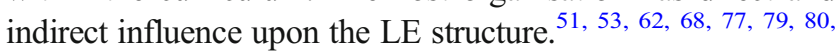
$85,86,90,91,102,104$

Direct influence of the host organisation upon the LE structure is primarily through the matching process and mentee and mentor training. ${ }^{49,56,86,88,99,102,107}$ The host organisation also directly defines and oversees compliance of the roles, responsibilities and code of conduct of tutors and learners. ${ }^{53}$, $56,99,102,107$

Indirect support of the LE structure is evident through the financial and administrative support provided to the programme, learners and tutors by the host organisation. ${ }^{53}$, 85, 90, 91, 102, 104 Administrative support influences the planning and scheduling of rotations, guides supervisory support of learners and tutors $49,85,88$ and regulates the learning relationship. $^{51,68,79,80,85,104}$

The host organisation is itself influenced by departmental policies and broader curricular considerations. ${ }^{68,77,86}$ Regnant education and healthcare systems which set system-wide standards of practice and codes of conduct employed in the curriculum also influence the host organisation and the roles it plays. ${ }^{77,86}$ The available support and capacity of the host organisation then impact the content and design of the formal curriculum.

Tutor. Nine papers discussed the reciprocal relationship between tutors and the LE structure. ${ }^{36,51,58,61,65,66,69,84}$, 85, 99 The tutor's abilities and skills in maintaining ${ }^{85}$ and monitoring ${ }^{66,84,85,99}$ a structured learner-centred approach and in providing appropriate and timely feedback ${ }^{58,61,65}$ and support $^{36,85}$ influence the LE structure. The tutor's abilities and skills also impact oversight of the learning relationship. ${ }^{36,}$ ${ }^{51,} 58$ Hudson (2004) and Mann (2001) believe that the tutor also affects the LE structure when adapting their educational approaches to meet the learner's needs. ${ }^{69,85}$

The LE structure influences tutors through the selection, training and matching process it adopts, underlining a reciprocal relationship between LE structure and tutors. ${ }^{36,51,58,61 \text {, }}$ $65,66,69,84,85,99$

Learner. The learner also influences and is influenced by the LE structure. ${ }^{66,85,93,103}$ Through the selection, training and matching process, the LE structure determines admission to the learning programme. The learner's skills, knowledge, ${ }^{85,93}$ personal circumstances (e.g. stressors in their private life, relationships with peers ${ }^{66}$ ), beliefs, motivations and "open mindedness $" 85$ influence interactions between learner and the tutor and organisation ${ }^{103}$ and impacts the efficacy of the learning process. ${ }^{66,85,93,103}$

Learning Relationship. Fourteen articles ${ }^{19}$, 71, 82, 83, 85, 90, 100, 108-114 discussed the learning relationship between tutor and learner. The LE structure impacts the learning relationship 


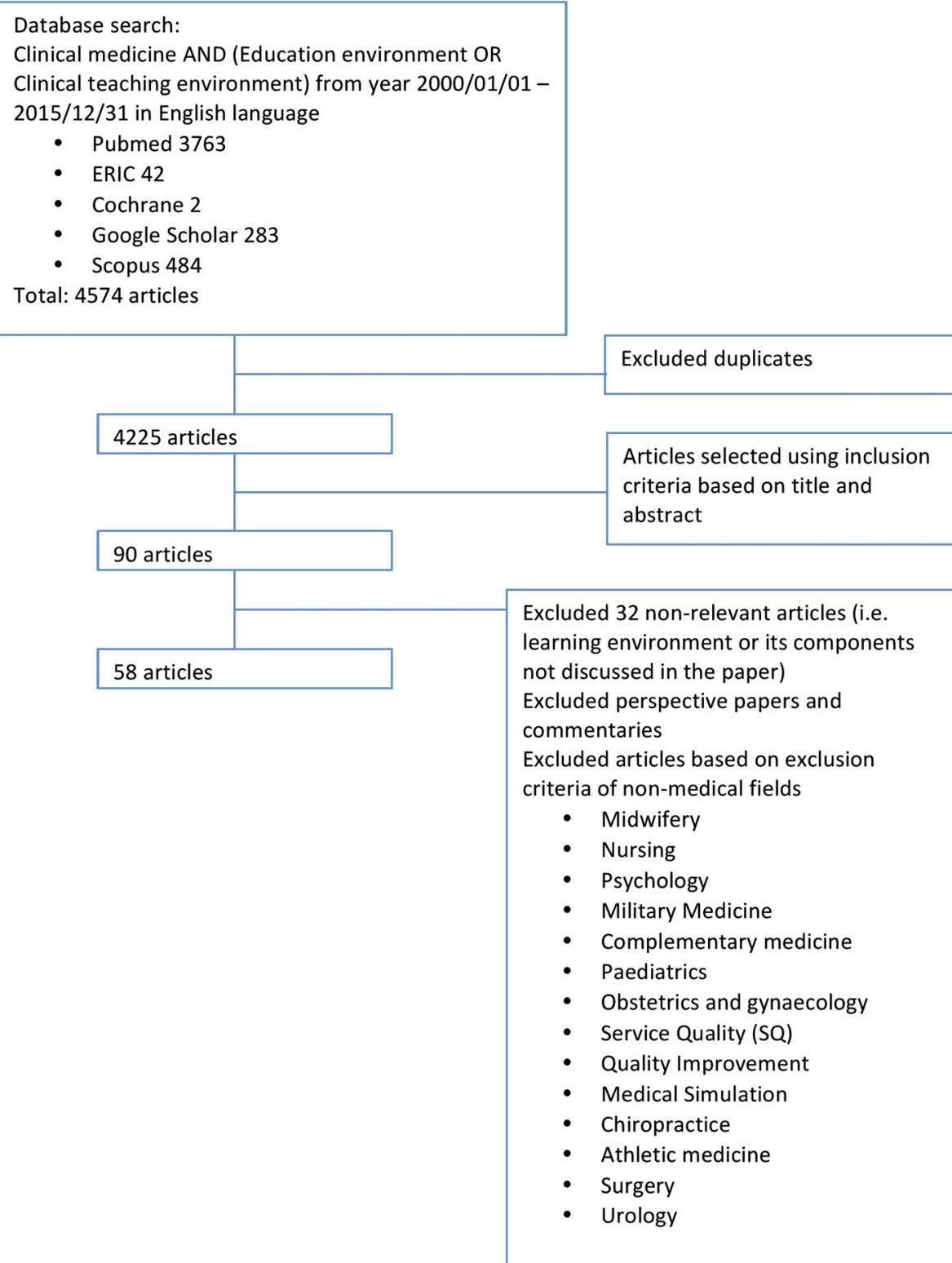

Figure 1 PRISMA chart.

through matching processes adopted, specifically by influencing the "goodness of fit" between learners and tutors or how well learners and tutors complement each other's personalities, work ethic and the presence of shared interests and goals. ${ }^{19,}{ }^{108-114}$ The LE structure also determines the form, content and frequency of interactions ${ }^{71,90,100}$ which impact the development of the learning relationship. ${ }^{71,90,100}$

Karani (2014) reveals that the learning relationship and the LE structure share a co-dependent relationship. Gan et al. (2014) expand upon this premise by suggesting that the relationship between the tutor and the learner and the LE structure are also affected by external factors and curricular influences. Adaptations to support the learning relationship affect the LE structure. $^{66,71}$ Gan et al. (2014) report that long hours, sleep deprivation and the need to adapt to changing environments impact learning relationships and thus the LE structure.

\section{Culture}

LE culture refers to the norms, values, beliefs, practices and support moulding the socioemotional environment in which learning occurs. ${ }^{105}$ There are five sub-themes to culture that include the informal and hidden curriculum, the host organisation, the tutor, the learner and the learning relationship (relationship between learner and tutor). The informal curriculum denotes "much of what occurs in clinical settings - the opportunistic, idiosyncratic, pop-up, and often unplanned instruction that takes place between anyone who is teaching (attendings, residents, other health care professionals) and trainees. The informal curriculum also takes place in nonclinical settings such as faculty offices, hallway interactions, or the countless other settings in which teachers and other health care providers interact with trainees". ${ }^{106}$ The hidden curriculum "includes the ideological and subliminal messages of both the 
formal and informal curricula. The hidden curriculum can be both human and structural; that is, it can be transmitted through human behaviours and through the structures and practices of institutions" 106 .

Informal and Hidden Curriculum. The hidden and informal curriculum affects learning ${ }^{88}$ and collaboration. ${ }^{36}$ The hidden and informal curriculum is shaped by the institution, tutors and learners and impacts learning through observation, reflection, ${ }^{71}$ and role modelling. ${ }^{79}$ Karani (2014) noted that residents employed "unwritten rules" and practices of the hospital environment to guide undergraduate learners navigating and adapting to the clinical environment.

Host Organisation. The host organisation directly and indirectly influences the LE culture. ${ }^{25,53,69,71,87,99}$ Ramani (2008) noted that the host organisation directly influences LE culture by "demystifying" the hidden curriculum. This may include specifying promotion criteria and clarifying the nature of academic tracks adopted by the programme. ${ }^{25}$ Indirect influences of the host organisation upon LE culture are apparent in the institutional vision, values and goals of its programmes and in the policies it adopts.

Tutor. Thirteen papers discussed the tutor's influence upon LE culture. $^{36,51,52,58,61,65,66,71,79,83-85,99}$ The tutor's skills at role modelling ${ }^{71}$ approachability, empathy, patience, collaboration, warmth, enthusiasm, respectfulness, commitment to teaching and the various aspects of professionalism inform practice and shape interactions with the learner. $^{36,52,66,79,80,83,85,99}$ Gan et al. (2014) reported that learners felt that informal "lessons" heavily influence students' emotions and behaviour.

Learner. The learner's skills, knowledge, ${ }^{85,} 93$ personal circumstances (e.g. stressors in their private life, relationships with peers ${ }^{66}$ ), beliefs, motivations and "open mindedness" $" 85$ also influence the learner's interactions with the tutor and organisation. ${ }^{103}$ A positive or negative personal and/or socioemotional climate alters the learner's perception of the learning environment. ${ }^{66}$

Learning Relationship. A collaborative $\mathrm{e}^{71,90,100}$ culture characterised by mutual commitment, authenticity, cooperation, honesty and flexibility and respect nurtures the learning relationship, creates personalised ties ${ }^{64,71,85,100}$ and prevents isolation of learners. ${ }^{66,}{ }^{94,}{ }^{101}$ Chinthammitr and Chierakul (2014) showed how good teacher-student relationships in Thai educational culture contributes to a positive learning environment. ${ }^{57}$ Young (2014) reported that a safe learning relationship allowed learners to test their limits and develop their professional identity whilst Mann (2001) noted that appreciation of the learning relationship motivated tutors. $^{85,103}$

\section{DISCUSSION}

This study dispenses with the notion of a dyadic relationship between the learner and tutor at the centre of the learning process and substitutes it with an entwined concept of the LE that involves the host organisation, the learner, the tutor, the relationships they share and the wider healthcare and educational systems (henceforth elements). Each element is interdependent and brings with it, its own goals, timelines, practices, code of conduct and expectations that creates a structure to the learning process referred to as the LE structure. Each element also brings with it individual norms, values and beliefs that come together to inform and influence interactions with one another creating the LE culture. ${ }^{105}$ LE structure maps out the learning process but is sufficiently flexible to contend

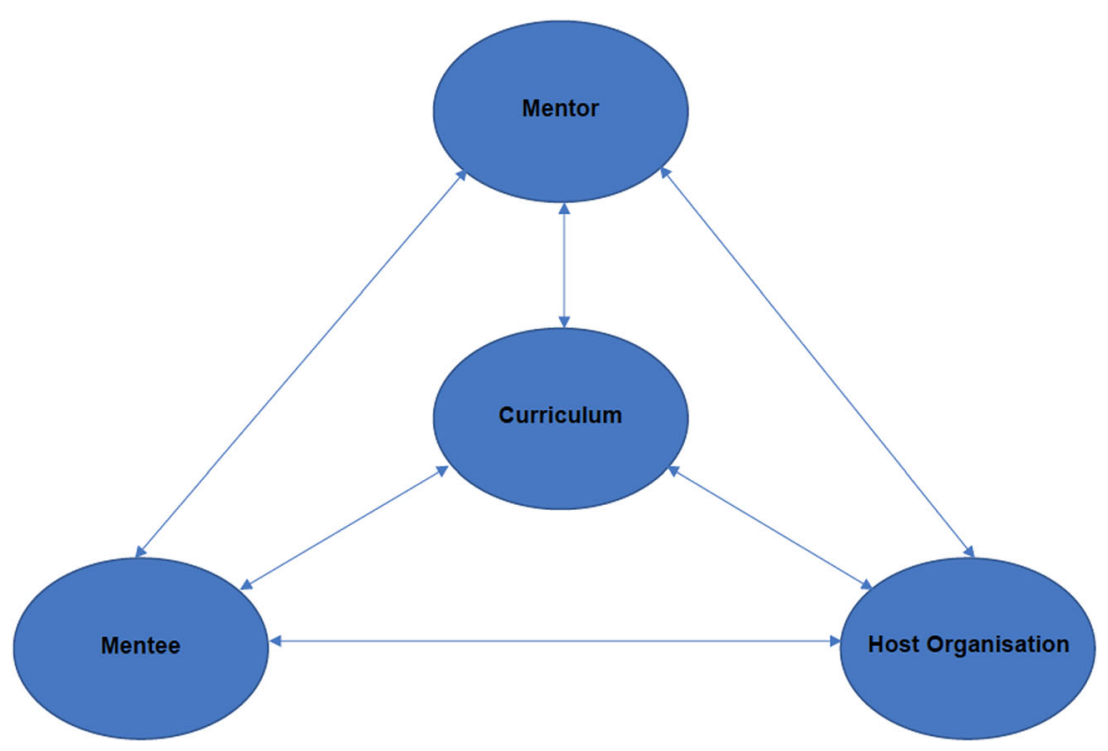

Figure 2 Interactions between the quartet of stakeholders. 
with the evolving needs of each element albeit within the confines of acceptable practice standards and guidelines. LE culture informs adaptations to the LE structure ensuring its personalization. The influence and co-dependence of elements within the LE culture and the LE structure underline the entwined, evolving, adapting, context-specific, goal-sensitive, learner-, tutor-, relationship- and host organisation-dependent nature of the LE (henceforth LE's nature). Concurrently, the presence of adaptations to the LE hints at the role of assessments of the learner's progress, abilities and needs by the tutor and evaluations of the progress of the programme by the host organisation. Assessments and evaluations within the mentoring process are neglected in prevailing data. ${ }^{3-9}$

\section{A Definition of LE}

New insights and data from this study suggest that LE may be defined as "the product of the culture and structure shaped by evolving interactions between the tutor, the learner, the host organization and the formal, informal and hidden curriculum (henceforth quartet of stakeholders). The structural element of the LE influences the quality of learning, supports the personal and professional development of the learner and the tutor and ensures that learning interactions remain within the confined accepted codes of conduct and professional standards of practice. Cultural considerations help personalize the LE and meet the unique and evolving needs of the quartet of stakeholders. These factors highlight LE's evolving, goal-sensitive, contextspecific, learner-, tutor-, relational-, organization-dependent nature (LE's nature). It forms part of the holistic evaluations to be considered when balancing the sometimes-competing demands for consistency and flexibility in the LE".

\section{Linking LEs and Mentoring Environments}

Commonalities in LE's and ME's overall goals and processes as well as their evolving, goal-sensitive, context-specific, mentee-, mentor- relational-, organisation-dependent nature support Pololi's (2002) and Kalén, Ponzer and Silén's (2012) posits that extrapolation of LE data to the ME setting is possible. ${ }^{3,7,29,30}$

In extrapolating LE data, MEs can be defined as "shaped by the nature, culture and structure of evolving mentoring relationships between the mentor, the mentee, the host organisation and the curriculum (henceforth quartet of stakeholders) (Fig. 2). MEs evolve to support and nurture mentoring relationships in dynamic conditions and as particular relationships amongst the quartet of stakeholders change. MEs influence the quality of education and professional practice."

\section{Limitations}

Whilst the themes identified in this review echo the key elements within prevailing tools to study learning environments such as the Dundee Ready Education Environment Measure (DREEM) ${ }^{39}, 115$ and the Postgraduate Hospital
Educational Environment Measure (PHEEM), ${ }^{116}$ the data comes from a small pool of articles published in English or with English translations. The presence of mainly North American and European accounts of LEs skews perspectives and raises questions as to the applicability of these findings in other healthcare settings given the importance of norms, values, beliefs and practices upon LEs.

Although there is overlap between ME and LE, and lessons gleaned from LE may inform the development of a conducive $\mathrm{ME}$, they require contextualisation to the local setting. Furthermore, many of the conclusions drawn remain rooted in "Cartesian reductionism and Newtonian principles of linearity" 117 which ignore the evolving and adaptive nature of LEs and MEs compromising the applicability of LE findings to MEs.

\section{CONCLUSION}

New insights into MEs provided here underline the need to contextualise, personalise and adapt mentoring programmes to local practice and individual mentees. Critical to this process is the need for effective assessment methods that move beyond "Cartesian reductionism and Newtonian principles of linearity"117 and can contend with ME's evolving nature. These evaluation tools must be robust, adaptable, holistic and longitudinal to account for the hitherto unrecognised influence of the host organisation, curriculum, healthcare and education systems. In addition, assessment methods are required to evaluate mentee progress, needs and abilities to better inform adaptations to the ME approach.

There is much to be understood of the mentoring process, not least in light of suggestions that the role and influence of each stakeholder is intertwined and requires careful scrutiny. We hope that our concept of ME expands upon prevailing ideas to better guide design and oversight of mentoring programmes and highlight the critical areas for much needed future research.

Acknowledgements: The authors wish to thank Ms. Annelissa Chin, Senior Librarian from Yong Loo Lin School of Medicine, National University of Singapore, for her guidance and advice on the literature search strategies for this paper. This paper is dedicated to the late Dr. $S$ Radha Krishna whose advice and insights were critical to conceptualization of this review.

Corresponding Author: Ying Pin Toh, MBBS, MRCPCH; Department of Family Medicine National University Hospital, 1E Kent Ridge Road, Singapore 119228, Singapore (e-mail: yingpintoh@gmail.com).

Compliance with Ethical Standards:

Conflict of Interest: The authors declare that they do not have a conflict of interest.

\section{REFERENCES}

1. Sambunjak D, Straus S, Marusic A. A Systematic Review of Qualitative Research on the Meaning and Characteristics of Mentoring in Academic Medicine. J Gen Intern Med 2009;25:72-78. 
2. Kashiwagi D, Varkey P, Cook D. Mentoring Programs for Physicians in Academic Medicine. Acad Med. 2013;88:1029-1037. Available at: https://doi.org/10.1097/acm.0b013e318294f368. Accessed 16 December 2018

3. Sng J, Pei Y, Toh Y, et al. Mentoring relationships between senior physicians and junior doctors and/or medical students: A thematic review. Med Teach 2017;39:866-875.

4. Toh YP, Lam B, Soo J, et al. Developing Palliative Care Physicians through Mentoring Relationships. Palliative Medicine \& Care: Open Access. 2017;4(1):1-9. https://doi.org/10.15226/2374-8362/4/1/ 00129. Accessed 16 December 2018.

5. Low C, Toh YL, Teo A, Toh YP, Krishna LKR. A narrative review of mentoring programs in General Practice. Educ Prim Care 2018. https://doi.org/10.1080/14739879.2018.1474723

6. Ikbal MFM, Wu JT, Wahab MT, Kanesvaran R, Krishna LKR Mentoring in Palliative Medicine: Guiding Program Design through Thematic Analysis of Mentoring in Internal Medicine between 2000 and 2015. J Palliat Care Med. 2017;07(05).

7. Tan B, Toh YL, Toh YP, et al. Extending Mentoring in Palliative Medicine-Systematic Review on Peer, Near- Peer and Group Mentoring in General Medicine. J Palliat Care Med. 2017;07(06).

8. Tan $\mathbf{Y}$, Teo $\mathbf{S}$, Pei $\mathbf{Y}$, et al. A framework for mentoring of medical students: thematic analysis of mentoring programmes between 2000 and 2015. Adv Health Sci Educ 2018;23(4):671-697.

9. Low CQT, Toh YL, Teo SWA et al. A narrative review of mentoring programmes in general practice. Educ Prim Care. 2018;1-9.

10. White H, Buhr G, Pinheiro S. Mentoring: A Key Strategy to Prepare the Next Generation of Physicians to Care for an Aging America. J Am Geriatr Soc 2009;57:1270-1277.

11. Hauer K, Teherani A, Dechet A, et al. Medical students' perceptions of mentoring: a focus-group analysis. Med Teach 2005;27:732-734

12. Loo TWW, Ikbal MFM, Wu JT, et al. Towards a Practice Guided Evidence Based Theory of Mentoring in Palliative Care. J Palliat Care Med. 2017;07.

13. Watson J, Clement D, Blom L, et al. Mentoring: Processes and Perceptions of Sport and Exercise Psychology Graduate Students. J Appl Sport Psychol 2009;21(2):231-246.

14. von der Borch P, Dimitriadis $\mathbf{K}$, Stormann $\mathbf{S}$, et al. A Novel Large scale Mentoring Program for Medical Students based on a Quantitative and Qualitative Needs Analysis. GMS Z Med Ausbild. 2011;28(2):Doc26.

15. Clark R, Harden S, Johnson W. Mentor Relationships in Clinical Psychology Doctoral Training: Results of a National Survey. Teach Psychol 2000;27(4):262-268.

16. Allen T, Eby L, Lentz E. The relationship between formal mentoring program characteristics and perceived program effectiveness. Pers Psychol 2006;59(1):125-153.

17. Arthur M, Kram K. Mentoring at Work: Developmental Relationships in Organizational Life. Adm Sci Q 1985;30(3):454.

18. Wahab MT, Wu JT, Ikbal MFM, et al. Creating Effective Interprofes sional Mentoring Relationships in Palliative Care- Lessons from Medicine, Nursing, Surgery and Social Work. J Palliat Care Med. 2016;06.

19. Snell LS, Frank JR. Competencies, the tea bag model, and the end of time. Med Teach 2010;32(8):629-630.

20. Davis O, Nakamura J. A Proposed Model for an Optimal Mentoring Environment for Medical Residents: A Literature Review. Acad Med 2010;85:1060-1066

21. Steele M, Fisman S, Davidson B. Mentoring and role models in recruitment and retention: A study of junior medical faculty percep tions. Med Teach 2012;35:e1130-e1138.

22. Straus $\mathbf{S}$, Chatur $\mathbf{F}$, Taylor $\mathbf{M}$. Issues in the Mentor-Mentee Relation ship in Academic Medicine: A Qualitative Study. Acad Med 2009;84: 135-139.

23. Lindgren $\mathbf{U}$. An empirical study of mentorship in higher education in Sweden [dissertation]. Sweden: Umeå University Department of Social Sciences at Umeå

24. Markakis K, Beckman H, Suchman A, et al. The Path of Professionalism. Acad Med 2000;75:141-149.

25. Ramani S. Twelve tips to promote excellence in medical teaching. Med Teach 2006;28:19-23.

26. Pinilla S, Pander T, von der Borch $\mathbf{P}$, et al. 5 years of experience with a large-scale mentoring program for medical students. GMS Z Med Ausbild. 2015;32(1)

27. Wu JT, Wahab MT, Ikbal MFM, et al. Toward an Interprofessional Mentoring Program in Palliative Care - A Review of Undergraduate and Postgraduate Mentoring in Medicine, Nursing, Surgery and Social Work. J Palliat Care Med. 2016;06.
28. Allen TD, Eby LT, O'Brien KE, et al. The state of mentoring research: A qualitative review of current research methods and future research implications. J Vocat Behav 2018;73(3):343-357.

29. Pololi LH, Knight SM, Dennis K, et al. Helping medical school faculty realize their dreams: an innovative, collaborative mentoring program. Acad Med 2002;77(5):377-384.

30. Kalén S, Ponzer S, Silén C. The core of mentorship: medical students' experiences of one-to-one mentoring in a clinical environment. Adv Health Sci Educ 2012;17(3):389-401.

31. Genn J. AMEE Medical Education Guide No. 23 (Part 1): Curriculum, environment, climate, quality and change in medical education-a unifying perspective. Med Teach 2001;23:337-344.

32. The Executive Council, The World Federation for Medical Education. International standards in medical education: assessment and accreditation of medical schools' - educational programmes. A WFME position paper. Med Educ. 1998;32:549-558.

33. Hutchinson L. $\mathrm{ABC}$ of learning and teaching: Educational environment. BMJ. 2003;326:810-812.

34. Report of the Standing Committee on Postgraduate Education (London). Good Practice in SHO Training. Londson Department of Health; 1991.

35. Shochet $\mathbf{R}$, Colbert-Getz $\mathbf{J}$, Levine $\mathbf{R}$, et al. Gauging Events That Influence Students' Perceptions of the Medical School Learning Environment. Acad Med 2013;88:246-252.

36. Ramani S, Leinster S. AMEE Guide no. 34: Teaching in the clinical environment. Med Teach 2008;30(4):347-64.

37. Genn J. AMEE Medical Education Guide No. 23 (Part 2): Curriculum, environment, climate, quality and change in medical education - a unifying perspective. Med Teach 2001;23:445-454.

38. Soemantri D, Herrera C, Riquelme A. Measuring the educational environment in health professions studies: A systematic review. Med Teach 2010;32:947-952.

39. Roff S, McAleer S, Harden R, et al. Development and validation of the Dundee Ready Education Environment Measure (DREEM). Med Teach 1997; 19:295-299.

40. Schönrock-Adema J, Heijne-Penninga M, van Hell E, et al. Necessary steps in factor analysis: Enhancing validation studies of educational instruments. The PHEEM applied to clerks as an example. Med Teach 2009;31:e226-e232.

41. Riquelme A, Herrera C, Aranis C, et al. Psychometric analyses and internal consistency of the PHEEM questionnaire to measure the clinical learning environment in the clerkship of a Medical School in Chile. Med Teach 2009;31:e221-e225.

42. Herrera C, Pacheco J, Rosso F, et al. Evaluation of the undergraduate educational environment in six medical schools in Chile. Rev Med Chil 2010;138(6):677-684.

43. Moher D, Liberati A. Tetzlaff $\mathbf{J}$, et al. Preferred reporting items for systematic reviews and meta-analyses: the PRISMA statement. BMJ. 2009;339(jul21 1):b2535.

44. Braun V, Clarke V. Using thematic analysis in psychology. Qual Res Psychol. 2006;3(2):77-101. https://doi.org/10.1191/ 1478088706qp063oa. Accessed 16 December 2018

45. Strand P, Sjöborg $\mathbf{K}$, Stalmeijer $\mathbf{R}$, et al. Development and psychometric evaluation of the Undergraduate Clinical Education Environment Measure (UCEEM). Med Teach 2013;35(12):1014-1026

46. Haig A. Dozier M. BEME Guide No. 3: Systematic searching for evidence in medical education-Part 2: Constructing searches. Med Teach. 2003:25(5):463-484. https://doi.org/10.1080/ 01421590310001608667 Accessed 16 December 2018.

47. Frei E, Stamm M, Buddeberg-Fischer B. Mentoring programs for medical students - a review of the PubMed literature 2000-2008. BMC Med Educ. 2010;10(1). https://doi.org/10.1186/1472-6920-10-32 Accessed 16 December 2018.

48. Abraham RR, Ramnarayan K, Pallath V, et al. Perceptions of academic achievers and under-achievers regarding learning environment of Melaka Manipal Medical College (Manipal campus), Manipal, India, using the DREEM Inventory. Southeast Asian J Med Educ 2008;2:4854

49. Al-Ayed IH, Sheik SA. Assessment of the educational environment at the College of Medicine of King Saud University, RiyadhEast Mediterr Health J 2008; 14(4):953-9.

50. Al-Mohaimeed A. Perceptions of the educational environment of a new medical school, Saudi Arabia. Int J Health Sci 2013;7(2):150-9.

51. Alshehri SA, Alshehri AF, Erwin TD. Measuring the Medical School Educational Environment: Validating an Approach from Saudi Arabia. Health Educ J 2012;71(5):553-564. 
52. Alzahrani HA. Bullying among medical students in a Saudi medical school. BMC Res Notes 2012;5:335.

53. Avalos G, Freeman C, Dunne F. Determining the quality of the medical educational environment at an Irish medical school using the DREEM inventory. Ir Med J 2007;100(7):522-5.

54. Belayachi J, Razine R, Boufars A, et al. Moroccan medical students' perceptions of their educational environment. J Educ Eval Health Prof 2015; $12: 47$.

55. Bennett D, Kelly M, O'Flynn S. Are the bigger hospitals better: DREEM on? Ir J Med Sci 2010;179(4):515-9.

56. Byszewski A, Hendelman W, McGuinty C, et al. Wanted: role modelsmedical students' perceptions of professionalism. BMC Med Educ 2012;12:115.

57. Chinthammitr Y, Chierakul N. Learning environment and resident achievement. J Med Assoc Thail 2014;97(12):1269-73.

58. Clapham M, Wall D, Batchelor A. Educational environment in intensive care medicine - Use of Postgraduate Hospital Educational Environment Measure (PHEEM). Med Teach 2007;29(6):e184-e191.

59. Colbert-Getz JM, Kim S, Goode VH, et al. Assessing medical students' and residents' perceptions of the learning environment: exploring validity evidence for the interpretation of scores from existing tools. Acad Med 2014;89(12):1687-93.

60. Dehghani M, Athar O, Ashourioun V, et al. A transitional curriculum for preparing medical students for internship, does it work? J Res Med Sci 2013;18(6):506-9.

61. Dreyer A, Gibbs A, Smalley S, et al. Clinical Associate students' perception of the educational environment at the University of the Witwatersrand, Johannesburg. Afr J Prim Health Care Fam Med. 2015;7(1)

62. Dyrbye LN, Thomas MR, Harper W, et al. The learning environment and medical student burnout: a multicentre study. Med Educ 2009;43(3):274-82.

63. Ebrahimi S, Kojuri J. Comparison of two educational environments in early clinical exposure program based on Dundee Ready Educational Environment Measure. J Adv Med Educ Prof 2013;1(1):36-37.

64. Emilia O, Bloomfield L, Rotem A. Replication of a clinical learning environment survey for junior medical officers: a study of medical students in an Indonesian hospital. Focus on Health Professional Education 2005;7(3):36.

65. Finn Y, Avalos G, Dunne F. Positive changes in the medical educational environment following introduction of a new systems-based curriculum: DREEM or reality? Curricular change and the Environment. Ir J Med Sci $2014 ; 183(2): 253-8$

66. Gan R, Snell L. When the learning environment is suboptimal: exploring medical students' perceptions of "mistreatment". Acad Med 2014;89(4):608-17.

67. Haidet P, Kelly PA, Bentley S, et al. Not the same everywhere. Patientcentered learning environments at nine medical schools. J Gen Intern Med 2006;21(5):405-9.

68. Hendelman W, Byszewski A. Formation of medical student professional identity: categorizing lapses of professionalism, and the learning environment. BMC Med Educ 2014;14:139.

69. Hudson JN, Buckley P. An Evaluation of Case-Based Teaching: Evidence for Continuing Benefit and Realization of Aims. Teaching with Problems and Cases. Adv Physiol Educ 2004;28(1):15-22.

70. Jalili M, Hejri SM, Ghalandari M, et al. Validating modified PHEEM questionnaire for measuring educational environment in academic emergency departments. Arch Iran Med 2014;17(5):372-377.

71. Karani R, Fromme HB, Cayea D, et al. How medical students learn from residents in the workplace: a qualitative study. Acad Med 2014;89(3):490-6.

72. Kashner TM, Henley SS, Golden RM, et al. Studying the effects of ACGME duty hours limits on resident satisfaction: results from VA learners' perceptions survey. Acad Med 2010;85(7):1130-9.

73. Kavukcu E, Burgazli KM, Akdeniz M, et al. Family medicine and sports medicine students' perceptions of their educational environment at a primary health care center in Germany: using the DREEM questionnaire. Postgrad Med 2012;124(5):143-50.

74. Kelly M, Bennett D, Muijtjens A, et al. Can less be more? Comparison of an 8-item placement quality measure with the 50-item Dundee Ready Educational Environment Measure (DREEM). Adv Health Sci Educ Theory Pract 2015;20(4):1027-32.

75. Khan AS, Akturk Z, Al-Megbil T. Evaluation of the learning environment for diploma in family medicine with the Dundee Ready Education Environment (DREEM) Inventory. J Educ Eval Health Prof 2010;7
76. Koohpayehzadeh J, Hashemi A, Soltani Arabshahi K, et al. Assessing validity and reliability of Dundee ready educational environment measure (DREEM) in Iran. Med J Islam Repub Iran 2014;28:60.

77. Koutsogiannou P, Dimoliatis ID, Mavridis D, et al. Validation of the Postgraduate Hospital Educational Environment Measure (PHEEM) in a sample of 731 Greek residents. BMC Res Notes 2015;8:734.

78. Laack TA, Newman JS, Goyal DG, et al. A 1-week simulated internship course helps prepare medical students for transition to residency. Simul Healthc 2010;5(3):127-32.

79. Lam WW, Fielding R, Johnston JM, et al. Identifying barriers to the adoption of evidence-based medicine practice in clinical clerks: a longitudinal focus group study. Med Educ 2004;38(9):987-97.

80. Latta $\mathbf{L}$, Tordoff $\mathbf{D}$, Manning $\mathbf{P}$, et al. Enhancing clinical skill development through an Ambulatory Medicine Teaching Programme: an evaluation study. Med Teach 2013;35(8):648-54.

81. Luciani E, Cerritelli F, Waters M, et al. Osteopathic student satisfaction and preparedness to practice: A comparative study. Int $\mathrm{J}$ Ostheopath Med 2014;17(1):28-37.

82. Macallan DC, Kent A, Holmes SC, et al. A model of clinical problembased learning for clinical attachments in medicine. Med Educ 2009;43(8):799-807.

83. Makhdoom N, Khoshhal KI, Algaidi S, et al. 'Blended learning' as an effective teaching and learning strategy in clinical medicine: A comparative cross-sectional university-based study. J Taibah Univ Med Sci. 2013;8(1):12-17.

84. Makhdoom NM. Assessment of the quality of educational climate during undergraduate clinical teaching years in the College of Medicine, Taibah University. J Taibah Univ Med Sci 2009;4(1):42-52.

85. Mann KV, Holmes DB, Hayes VM, et al. Community family medicine teachers' perceptions of their teaching role. Med Educ 2001;35(3):27885.

86. Mladenovic J, Shea JA, Duffy FD, et al. Variation in internal medicine residency clinic practices: assessing practice environments and quality of care. J Gen Intern Med 2008;23(7):914-20.

87. Mojaddidi MA, Khoshhal KI, Habib F, et al. Reassessment of the undergraduate educational environment in College of Medicine, Taibah University, Almadinah Almunawwarah, Saudi Arabia. Med Teach 2013;35 Suppl 1:S39-46

88. Mowafy M, Zayed M, Eid N. The Effect of Family Medicine Programs' Educational Environment on Post graduate Medical Students' Learning Perceptions, in Egypt. Egyptian Journal of Community Medicine. 2015;33(4).

89. Naghizadeh Moogari Z, Koohpayehzadeh J, Roff S, et al. Psychometric analysis of the ambulatory care learning education environment measure (ACLEEM) in Iran. Med J Islam Repub Iran 2015;29:299.

90. Ottenheijm RP, Zwietering PJ, Scherpbie AJ, et al. Early studentpatient contacts in general practice: an approach based on educational principles. Med Teach 2008;30(8):802-8.

91. Pales J, Gual A, Escanero J, et al. Educational climate perception by preclinical and clinical medical students in five Spanish medical schools. Int J Med Educ 2015;6:65-75.

92. Reid WA, Duvall E, Evans P. Can we influence medical students' approaches to learning? Med Teach 2005;27(5):401-7.

93. Reid WA, Evans P, Duvall E. Medical students' approaches to learning over a full degree programme. Med Educ Online 2012;17.

94. Seabrook MA. Clinical students' initial reports of the educational climate in a single medical school. Med Educ 2004;38(6):659-69.

95. Shankar PR, Bharti R, Ramireddy R, et al. Students' perception of the learning environment at Xavier University School of Medicine, Aruba: a follow-up study. J Educ Eval Health Prof 2014;11.

96. Shankar PR, Dubey AK, Balasubramanium R. Students' perception of the learning environment at Xavier University School of Medicine, Aruba. J Educ Eval Health Prof 2013;10.

97. Smith CF, Mathias HS. Medical students' approaches to learning anatomy: students' experiences and relations to the learning environment. Clin Anat. 2010;23(1):106-14

98. Tokuda Y, Goto E, Otaki J, et al. Undergraduate educational environment, perceived preparedness for postgraduate clinical training, and pass rate on the National Medical Licensure Examination in Japan. BMC Med Educ 2010;10:35.

99. Tsai JC, Chen CS, Sun IF, et al. Clinical learning environment measurement for medical trainees at transitions: relations with sociocultural factors and mental distress. BMC Med Educ 2014;14:226. 
100. Vaughan B, Carter A, Macfarlane C, et al. The DREEM, part 1: measurement of the educational environment in an osteopathy teaching program. BMC Med Educ 2014; 14:99.

101. Vieira JE, Do Patrocínio Tenório Nunes M, De Arruda Martins M. Directing student response to early patient contact by questionnaire. Med Educ 2003;37(2):119-125.

102. Widyandana D, Majoor GD, Scherpbier AJ. Comparison of three clinical environments for pre-clinical clinical skills training. Med Teach 2011;33(11):928-32.

103. Young JE, Williamson MI, Egan TG. Students' reflections on the relationships between safe learning environments, learning challenge and positive experiences of learning in a simulated GP clinic. Adv Health Sci Educ Theory Pract 2016;21(1):63-77.

104. Zumberg MS, Broudy VC, Bengtson EM, et al. Preclinical Medical Student Hematology/Oncology Education Environment. J Cancer Educ 2015;30(4):711-718.

105. Watling C. When I say ... learning culture. Med Educ 2015;49:556-557.

106. Wear D, Skillicorn J. Hidden in Plain Sight: The Formal, Informal, and Hidden Curricula of a Psychiatry Clerkship. Acad Med 2009;84:451458 .

107. Hafferty F. Beyond curriculum reform. Acad Med 1998;73:403-7.

108. Carraccio C, Wolfsthal S, Englander R, et al. Shifting Paradigms. Acad Med 2002;77:361-367.

109. McGaghie WC, Miller GE, Sajid A, et al. Competency-based curriculum development in medical education. Public Health Pap 1978;(68):1191.
110. Iobst W, Sherbino J, Cate $\mathbf{O}$, et al. Competency-based medical education in postgraduate medical education. Med Teach 2010;32:651-656.

111. Harris $\mathbf{P}$, Snell $\mathbf{L}$, Talbot $\mathbf{M}$, et al. Competency-based medical education: implications for undergraduate programs. Med Teach 2010;32:646-650.

112. Frank JR, Snell LS, Cate OT, et al. Competency-based medical education: theory to practice. Med Teach 2010;32(8):638-645.

113. Holmboe E, Ward D, Reznick R, et al. Faculty Development in Assessment: The Missing Link in Competency-Based Medical Education. Acad Med 2011;86:460-467.

114. Holmboe ES, Sherbino J, Long DM, et al. The role of assessment in competency-based medical education. Med Teach 2010;32(8):676-682.

115. Miles S, Swift L, Leinster S. The Dundee Ready Education Environment Measure (DREEM): A review of its adoption and use. Med Teach 2012;34:e620-e634

116. Roff S, McAleer S, Skinner A. Development and validation of an instrument to measure the postgraduate clinical learning and teaching educational environment for hospital-based junior doctors in the UK. Med Teach 2005;27:326-331.

117. Mennin S. Self-organisation, integration and curriculum in the complex world of medical education. Med Educ 2010;44:20-30.

Publisher's Note Springer Nature remains neutral with regard to jurisdictional claims in published maps and institutional affiliations. 